\title{
Development of microsatellite markers for the kelp grouper Epinephelus bruneus by 454 pyrosequencing and transfer to related species
}

\author{
J.-H. Kang ${ }^{1}$, S.G. Yang' ${ }^{2}$, T.S. Moon ${ }^{2}$, J.-Y. Park ${ }^{1}$ and T.-J. Choi ${ }^{3}$ \\ ${ }^{1}$ Biotechnology Research Division, \\ National Fisheries Research and Development Institute, \\ Busan, Republic of Korea \\ ${ }^{2}$ Future Aquaculture Research Center, \\ National Fisheries Research and Development Institute, \\ Jeju, Republic of Korea \\ ${ }^{3}$ Department of Microbiology, Pukyong National University, \\ Busan, Republic of Korea \\ Corresponding author: T.-J. Choi \\ E-mail: choitj@pknu.ac.kr
}

Genet. Mol. Res. 12 (4): 5485-5493 (2013)

Received March 27, 2013

Accepted October 22, 2013

Published November 13, 2013

DOI http://dx.doi.org/10.4238/2013.November.13.1

\begin{abstract}
The kelp or longtooth grouper (Epinephelus bruneus), which inhabits Eastern Asia, is the most economically important of 11 grouper species that inhabit the Southern Sea near Jeju Island in Korea. This species is listed as vulnerable by the International Union for the Conservation of Nature and Natural Resources because of a rapid decrease in its resources. We developed microsatellite markers for E. bruneus using the pyrosequencing technique for applications in resource management and aquaculture. In addition, we tested the cross-species transferability of the microsatellite markers in four species belonging to the Epinephelus genus. Among 66,452 simple sequence repeats, 64 loci containing more than eight CA or TG repeats were randomly selected for primer synthesis; 45 primer sets $(75.0 \%)$ produced polymerase chain reaction (PCR) products of 100 -
\end{abstract}


$300 \mathrm{bp}$ and were selected as candidates. After primary testing with four $E$. bruneus fish, 28 polymorphic loci were selected as the final microsatellite markers, and 23 sets showing clear amplification of polymorphic loci were used to analyze 71 fish. These loci have allele numbers ranging from 2 to 23. Null alleles were detected at three loci, and three loci showed an excess of homozygotes in the Hardy-Weinberg equilibrium test. Of the three species used for cross-species transfer of these markers, Epinephelus moara showed the highest transferability $(92.9 \%)$ and polymorphism $(67.9 \%)$, followed by Epinephelus fuscoguttatus (75.0 and 67.9\%, respectively) and Epinephelus septemfasciatus (57.1 and 46.4\%, respectively). These results suggested that these microsatellite loci should be valuable tools for population genetic studies of the species Epinephelus.

Key words: Kelp grouper; Pyrosequencing; Cross-species; Transferability

\section{INTRODUCTION}

The common name grouper is usually given to fish in one of two genera, Epinephelus and Mycteroperca, in the subfamily Epinephelinae of the family Serranidae in the order Perciformes (Heemstra and Randall, 1993). Because of their high prices and good meat quality, groupers are regarded as a new aquaculture species in East and Southeast Asia, and artificial larval rearing of these species is practiced today (Marte, 2003). Among the groupers, Epinephelus bruneus (Bloch), the kelp grouper or longtooth grouper, is a sedentary marine fish that lives in rocky areas of shallow coastal regions and in some deeper areas. It is native to Eastern Asia, including China, Hong Kong, Japan, Korea, the Republic of the Philippines, Taiwan, and Vietnam (Choi et al., 2002).

E. bruneus is vulnerable to a wide range of gear types, including long-liners, trawlers, and hand-liners. Notably, bottom trawling within its habitat has been a threat to both juveniles and adults (Chan, 1968), resulting in a severe decrease in the species' natural resources and causing it to be listed as a vulnerable species in the International Union for the Conservation of Nature and Natural Resources (IUCN) Red List of Threatened Species (Thierry et al., 2008).

Among 11 species of grouper inhabiting the Southern Sea near Jeju Island in Korea, E. bruneus is the most important and is a very expensive marine fish. Despite recent success in the small-scale production of larvae and juveniles, the aquaculture of E. bruneus in Korea and other countries remains problematic because of the high mortality during the early life stages of artificially reared larvae (Sawada et al., 1999; Song et al., 2005).

Understanding the genetic diversity of natural and brood stocks is necessary for future management of natural resources, restoration by releasing artificially produced juveniles, and production of high-quality larvae and juveniles for aquaculture. However, DNA markers suitable for the identification of individual fish and for assessing parentage and genetic relationships in this species are limited.

Among the diverse molecular makers used for the analysis of genetic diversity and population structure, the microsatellite maker, also known as simple sequence repeats (SSR), is one of the most efficient molecular tools for determining parentage, inferring genetic structure and gene flow patterns, and assessing the origins of introduced populations because of 
its polymorphic characteristics, co-dominant inheritance, and high reproducibility (Jarne and Lagoda, 1996; Chistiakov et al., 2006).

Traditional methods for the isolation of microsatellite DNA loci that rely on tedious, labor-intensive, and expensive processes involving the screening of genomic libraries using repetitive probes and sequencing positive clones are now being replaced by next-generation sequencing (NGS) technologies. NGS employs rapid and cost-effective parallel processing of millions of templates and can produce gigabases of sequence data in a single run. Individual reads or assembled contigs are sufficiently long $(>500 \mathrm{bp})$ to capture individual microsatellites and their flanking sequences for polymerase chain reaction (PCR) primer design. The large numbers of random sequence reads that contain many thousands of microsatellite markers eliminate the enrichment process used in traditional methods (Hamilton et al., 1999; Zane et al., 2002; Kircher and Kelso, 2010). The other advantage of NGS methods is the high crossspecies transferability of the developed makers, which is due to the large number of candidates that can be tested in related species and has been proven to be effective in several marine species (Saarinen and Austin, 2010; Greenley et al., 2011; Kang et al., 2012).

In this study, we developed microsatellite markers for the economically important endangered species $E$. bruneus for future application in resource management and aquaculture. In addition, we tested the cross-species transferability of the developed microsatellite markers in four species belonging to the Epinephelus genus.

\section{MATERIAL AND METHODS}

\section{Samples and DNA preparation}

A total of 71 wild E. bruneus samples were collected from the Southern Sea near Jeju Island. Muscle tissue samples were preserved in $100 \%$ ethanol at the sampling site and were then transported to the laboratory for DNA extraction. Total DNA was isolated from each sample using a MagExtractor MFX-6100 automated DNA extraction system (Toyobo, Osaka, Japan). The extracted genomic DNA was quantified using a Nanodrop ND-1000 spectrophotometer (Thermo Fisher Scientific, Barrington, IL, USA) and was stored at $-20^{\circ} \mathrm{C}$ prior to genomic DNA pyrosequencing. Four fish were tested for primer performance, and eight fish were used in the analysis of polymorphisms. For cross-species transferability testing, DNA was extracted by the same method from ethanol-fixed tissues from four related species belonging to the same genus that had been stored at the National Fisheries Research and Development Institute in Busan, Korea.

\section{DNA sequencing}

DNA sequencing was conducted by 454 pyrosequencing using a Genome Sequencer FLX-454 System (GS FLX sequencer; 454 Life Sciences, Branford, CT, USA) with a Pico TiterPlate (454 Life Sciences). Purified DNA was sheared three times and used for library construction. Sample preparation and DNA sequencing were performed according to manufacturer instructions (Roche Diagnostics, Mannheim, Germany). Sequencing was conducted at the National Instrumentation Center for Environmental Management (Seoul National University, Seoul, Korea). Sequences with over 600 base pairs (bps) were selected for analysis, and the quality scores of the sequences were maintained over 30. 


\section{Sequence assembly and SSR identification}

The raw data from the sequencer system were assembled using the Newbler 2.6 software. To search for SSRs in the genomic sequence, the assembled contig consensus sequences and the unassembled singleton sequences were merged. The sequence was filtered for high-quality scores using Less Useful Chunks Yank (LUCY) 1.20p, and a modified SSR finder.pl perl program was used to find SSRs with dinucleotide and trinucleotide repeats. $\overline{\mathrm{A}}$ total of 176 SSRs containing CA, CT, the or AT repeats were randomly selected, and a primer set flanking each SSR was designed using the Primer3 software (available at http://biotools. umassmed.edu/bioapps/primer3_www.cgi). Basic local alignment search tool (available from the National Center for Biotechnology Information) was used to test for primer redundancy using an e-value cutoff of $\leq 0.001$.

\section{PCR and genotyping}

The performance of the 64 designed primer sets was tested using DNA from four $E$. bruneus fish. The PCR products were electrophoresed on a $1.5 \%$ agarose gel, and 45 primer sets $(75.0 \%)$ yielded PCR products of 100-300 bp. Thirty of these primer sets were labeled and used to amplify DNA from eight fish. The forward primer of each pair was 5'-end labeled with the fluorescent dyes 6-FAM, NED, and HEX (Life Technologies, Carlsbad, CA, USA). PCR was performed in a $10-\mu \mathrm{L}$ reaction mixture containing $0.25 \mathrm{U}$ Ex Taq DNA polymerase (Takara Biomedical, Inc., Shiga, Japan), 1X PCR buffer, $0.2 \mathrm{mM}$ dNTPs, 10 pmol of each primer, and 100 ng template DNA using a PTC 200 DNA Engine (MJ Research, Waltham, MA, USA). The PCR conditions were as follows: $11 \mathrm{~min}$ at $95^{\circ} \mathrm{C} ; 35$ cycles of $1 \mathrm{~min}$ at $94^{\circ} \mathrm{C}$, $1 \mathrm{~min}$ at $60^{\circ} \mathrm{C}$, and $1 \mathrm{~min}$ at $72^{\circ} \mathrm{C}$; and a final extension of $5 \mathrm{~min}$ at $72^{\circ} \mathrm{C}$. Microsatellite polymorphisms were detected using an ABI PRISM 3130 XL Automated DNA Sequencer (Applied Biosystems, Foster City, CA, USA), and alleles were designated according to PCR product size relative to a molecular size marker (GeneScan 400 HD ROX; Life Technologies).

\section{Statistical analysis}

The number of alleles per locus, allele frequency, and heterozygosity were calculated using Arlequin 3.0. Tests for population-wide linkage disequilibrium between pairs of loci and deviations from Hardy-Weinberg equilibrium (HWE) were estimated using GENEPOP (ver. 4.0; http://kimura.univ-montp2.fr/ rousset/Genepop.htm), and the adjusted P values for both analyses were obtained using a sequential Bonferroni test for multiple comparisons. MICROCHECKER version 2.2.3 was used to test for the presence of null alleles.

\section{RESULTS}

\section{Pyrosequencing and characteristics of identified repeats}

NGS produced 684,929 reads of a total of $355,503,621 \mathrm{bp}$. The average read length was $519 \mathrm{bp}$. A total of 6928 reads, encompassing 3,794,761 bp and with an average length of $547 \mathrm{bp}$, were constructed by Newbler version 2.6. In addition, we found 572,445 unassembled 
singleton sequences. The total number of reads after trimming low-quality sequences was 563,307 . These reads had a combined length of 305,874,669 bp and were used in the search for SSRs. The numbers of reads with dinucleotide repeats were 7163 for AT, 40,731 for CA, 18,220 for CT, and 338 for GC repeats. The sequences and numbers of 15,927 trinucleotide repeats are shown in Table 1. From the 66,452 SSRs containing dinucleotide repeats, 64 loci containing more than eight CA or TG repeats were randomly selected for primer synthesis. The primer sets were tested in four E. bruneus fish, and 45 primer sets $(75.0 \%)$ produced PCR products of 100-300 bp. Among these, 28 sets $(62.2 \%)$ showed clear amplification, and those with an annealing temperature of $60^{\circ} \mathrm{C}$ were selected for further analysis. Detailed information about these primers and their fluorescent labeling is shown in Table 2.

\begin{tabular}{|c|c|c|c|}
\hline \multicolumn{2}{|c|}{ Primary sequence data } & \multicolumn{2}{|l|}{ No. } \\
\hline \multicolumn{2}{|c|}{ Total No. of reads } & \multicolumn{2}{|l|}{684,929} \\
\hline \multicolumn{2}{|c|}{ Total No. of bases } & \multicolumn{2}{|l|}{$355,503,621$} \\
\hline \multicolumn{2}{|c|}{ No. of contigs } & \multicolumn{2}{|l|}{6,928} \\
\hline \multicolumn{2}{|l|}{ No. of bases } & \multicolumn{2}{|l|}{$3,794,761$} \\
\hline \multicolumn{2}{|l|}{ No. of singleton } & \multicolumn{2}{|l|}{572,445} \\
\hline \multicolumn{2}{|c|}{ Total No. of reads after trimmed } & \multirow{2}{*}{\multicolumn{2}{|c|}{$\begin{array}{l}563,307 \\
305,874,669\end{array}$}} \\
\hline \multicolumn{2}{|c|}{ Total No of read-length after trimmed } & & \\
\hline Di-nucleotide & No. & Di-nucleotide & No. \\
\hline AT & 7,163 & СТ & 18,220 \\
\hline $\mathrm{CA}$ & 40,731 & GC & 338 \\
\hline Tri-nucleotide & No. & Tri-nucleotide & No. \\
\hline AAA & 11 & TAC & 163 \\
\hline AAT & 1,284 & TTG & 745 \\
\hline AAG & 547 & TTC & 605 \\
\hline AAC & 748 & TGA & 574 \\
\hline ATA & 869 & TGG & 350 \\
\hline ATG & 509 & TGC & 722 \\
\hline ATC & 434 & TCG & 11 \\
\hline AGA & 770 & TCC & 811 \\
\hline AGT & 196 & GAG & 1,192 \\
\hline AGG & 797 & $\mathrm{GAC}$ & 21 \\
\hline $\mathrm{AGC}$ & 840 & GTG & 265 \\
\hline $\mathrm{ACA}$ & 674 & GGG & 1 \\
\hline ACG & 17 & GGC & 44 \\
\hline $\mathrm{ACC}$ & 217 & GCG & 35 \\
\hline TAA & 1,068 & CAG & 1,240 \\
\hline TAG & 140 & CGG & 27 \\
\hline
\end{tabular}

\section{Genetic variability in microsatellite loci}

Among the 28 primers sets tested, 23 sets showing clear amplification of polymorphic loci and five showing amplification of monomorphic loci were used for PCR analysis of 71 individuals. These loci have allele numbers ranging from 2 to 23 (mean 6.7). Null alleles were detected at the Eb02-nfrdi, Eb32-nfrdi, and Eb63-nfrdi loci. Three loci, Eb02-nfrdi, Eb42-nfrdi, and Eb63-nfrdi, showed deviation from HWE with greater expected heterozygosity $\left(H_{\mathrm{E}}\right)$ values than observed heterozygosity $\left(H_{\mathrm{O}}\right)$ values, indicating that the excess of homozygotes was probably due to the presence of the null alleles at the Eb02-nfrdi and Eb63-nfrdi loci. This was also shown by the significant positive $F_{\text {IS }}$ values at four loci: Eb02-nfrdi, Eb42-nfrdi, Eb32-nfrdi, and Eb63-nfrdi (Table 2). Nine loci showed negative $F_{\text {IS }}$ values, but they were not statistically significant $(\mathrm{P}<0.05)$. 
Table 2. Characteristics of Epinephelus bruneus microsatellite loci.

\begin{tabular}{|c|c|c|c|c|c|c|c|}
\hline Locus & Forward primer sequence $\left(5^{\prime} \rightarrow 3^{\prime}\right)$ & Reverse primer sequence $\left(5^{\prime} \rightarrow 3^{\prime}\right)$ & Motif & $\begin{array}{l}\text { No. of } \\
\text { alleles }\end{array}$ & $H_{\mathrm{o}}$ & $H_{\mathrm{E}}$ & $F_{\text {IS }}$ \\
\hline 001-nfrdi & NED GAGGGAAGACAGCCCTAGTAGT & AACGATTTGATTACGGAATTG & 10 & 4 & 0.310 & 0.324 & 0.044 \\
\hline Eb02-nfrdi & 6-FAM TGTTTAGTTTGTTTGGTTATTGTCA & AGTTCTCTGTTGTCAGCCATTT & $(\mathrm{TG})_{9}$ & 3 & 0.394 & 0.555 & $0.289 *$ \\
\hline Eb05-nfrdi & NED GATCAACTTCCGACTTTCTTTG & CCCACACATCATAAAACACTCA & $(\mathrm{TG})_{14}$ & 6 & 0.732 & 0.750 & 0.023 \\
\hline Eb06-nfrdi & HEX GTTTGACTCACTCACTCCCTTC & ITAAGAGCGTACTGAGCTGGTT & $(\mathrm{CA})_{14}$ & 12 & 0.761 & 0.719 & -0.059 \\
\hline Eb08-nfrdi & NED ATCTTGTGGAGCCTTTTAGTGA & CAGGAAATAAGACAGGAAGCAC & $(\mathrm{TG})_{17}$ & 5 & 0.366 & 0.308 & -0.187 \\
\hline Eb11-nfrdi & HEX CCGCTTTTTACACTACGAGTC & ITCCTCGAGCAAGATACTGAAC & $(\mathrm{CA})_{10}$ & 1 & - & - & - \\
\hline Eb22-nfrdi & HEX AAGCAAAAAGCTTTGAGAGTTG & ATACACACGTGGACAGCAGAC & $(\mathrm{TG})_{12}$ & 7 & 0.775 & 0.800 & 0.031 \\
\hline Eb24-nfrdi & HEX CTGACTTGCTCGTATACTGCTG & AGCAATTCCACTCTGAGAGAAC & $(\mathrm{TG})_{9}$ & 4 & 0.338 & 0.358 & 0.054 \\
\hline Eb27-nfrdi & HEX TATCTCCTTCCTTCATGCAGAT & GAGTGTTACCTGCTCTTGTTCC & $(\mathrm{CA})_{11}$ & 3 & 0.085 & 0.109 & 0.222 \\
\hline Eb28-nfrdi & NED CATAGCAGTGGTTGAGAAACAA & CTTCAAACATGGATCAAGACAA & $(\mathrm{CA})_{12}$ & 23 & 1.000 & 0.943 & -0.061 \\
\hline Eb32-nfrdi & HEX AGCTGAACCATGCAGTAGAAAT & GGTCAGGAAAACAAGACAAGAG & $(\mathrm{CA})_{14}$ & 6 & 0.457 & 0.629 & $0.273 *$ \\
\hline Eb33-nfrdi & NED GAAACTTGATCATGACATCCCT & ACААССТСТСАСАСТССТСАТС & $(\mathrm{TG})_{8}$ & 3 & 0.028 & 0.028 & -0.004 \\
\hline Eb40-nfrdi & 6-FAM TGAGTGCACACAATGTGAATAA & ACGGTGTCCTGTAAATGTCTTC & $(\mathrm{TG})_{11}^{8}$ & 1 & - & - & - \\
\hline Eb42-nfrdi & NED GATTAGCTGCTTAATGAGCCTG & GAGAGAGTGTTTAGGAGCTGGA & $(\mathrm{CA})_{17}$ & 7 & 0.577 & 0.634 & $0.089 *$ \\
\hline Eb45-nfrdi & HEX ATCTACTCTGTCGGCACAAGAT & CATAGTCCACCTGAAAGGTCAT & $(\mathrm{TG})_{13}$ & 16 & 0.704 & 0.752 & 0.063 \\
\hline Eb46-nfrdi & HEX TGCCTCATACATTTCAAAAAGA & AAATATACAACCGCCACTGTTC & $(\mathrm{TG})_{13}$ & 1 & - & - & - \\
\hline Eb47-nfrdi & CATGC & $\mathrm{GCA}$ & $(\mathrm{CA}$ & 1 & - & - & - \\
\hline Eb49-nfrdi & NED GC & $\mathrm{C}$ & $(\mathrm{CA})_{9}^{8}$ & 2 & 0.479 & 0.431 & -0.112 \\
\hline Eb50-nfrdi & 6-FAM C & TGTGCATGTGTGT & $(\mathrm{CA})_{17}$ & 8 & 0.803 & 0.798 & $-0.006^{*}$ \\
\hline Eb51-nfrdi & 6-FAM CTCACCACATCACTGCAAATAC & AAATTGCAGTTCATCATTAAAGG & $(\mathrm{TG})_{8}$ & 1 & - & - & - \\
\hline Eb52-nfrdi & 6-FAM ACCTAAAACACCCAGACAACAC & CAAACGCGTATATTGTCAGCTA & $(\mathrm{CA})_{9}^{8}$ & 3 & 0.352 & 0.315 & -0.117 \\
\hline Eb56-nfrdi & HEX CATGTTGGTTCGTGTCTGTTAG & TGCTTTTCTCTTTTCCTCTCTG & $(\mathrm{TG})_{12}$ & 5 & 0.423 & 0.418 & -0.010 \\
\hline Eb57-nfrdi & 6-FAM CAACAGTGTGAACCAAAACAAT & ACCATGTCTTTGTTGCTGCT & $(\mathrm{CA})_{19}$ & 7 & 0.493 & 0.593 & 0.169 \\
\hline Eb58-nfrdi & NED CATTCAGAGCATTAATATCGCA & TCCCAAAATCAGCTATAACCAC & $(\mathrm{TG})_{10}$ & 3 & 0.507 & 0.530 & 0.043 \\
\hline Eb59-nfrdi & NED GTCCTTTACCTCTCCTTACCGT & AGCCTGTAAATACTCTCCСТCC & $(\mathrm{CA})_{12}$ & 3 & 0.507 & 0.592 & 0.144 \\
\hline Eb60-nfrdi & 6-FAM TGTCAAATTATCAACAAGCCAG & TATGTTGACTTTGGGAAGCTCT & $(\mathrm{C}$ & 16 & 0.873 & 0.874 & 0.000 \\
\hline Eb63-nfrdi & 6-FAM TGTGTGCAGTAAAAGAAAGCAT & CACTTCACCTGAGAGTGACAAA & (Tc & 3 & 0.235 & 0.510 & $0.539^{*}$ \\
\hline Eb64-nfrdi & 6-FAM ATGTTCACTTTTCTCGTCCTGT & CTGTCAGGTTCGTAGACAGAAA & $(\mathrm{TG})_{13}$ & 4 & 0.543 & 0.531 & -0.023 \\
\hline
\end{tabular}

$H_{\mathrm{O}}$ and $H_{\mathrm{E}}=$ observed and expected heterozygosities; $F_{\mathrm{IS}}=$ inbreeding coefficient. ${ }^{*} F_{\mathrm{IS}}=$ significant deviation from Hardy-Weinberg equilibrium $(\mathrm{P}<0.05)$.

\section{Cross-species amplification}

Cross-species amplification of the 23 polymorphic loci and five additional monomorphic loci whe conducted in three related species in the same genus (Epinephelus). As shown in Table 3, two of the five monomorphic loci were not amplified from any of the species tested, and the other three were polymorphic in at least one species tested (60\% transferability and polymorphism). In contrast, among the 23 polymorphic loci, Eb01-nfrdi was not amplified from any of the species tested. The Eb02-nfrdi and Eb33-nfrdi loci, which were polymorphic in E. bruneus, showed monomorphic amplification in one and two species tested, respectively ( $95.6 \%$ transferability and $87.0 \%$ polymorphism). Among the three species tested, E. moara showed the highest transferability ( $92.9 \%$ ) and polymorphism (67.9\%), followed by $E$. fuscoguttatus (75.0 and $67.9 \%$ transferability and polymorphism, respectively) and $E$. septemfasciatus (57.1 and 46.4\% transferability and polymorphism, respectively).

\section{DISCUSSION}

The rapid decrease in the natural resource represented by the highly valued E. bruneus made it a target species for aquaculture in Japan and Korea (Kato et al., 2004). One major constraint in the development of grouper aquaculture is that, like other grouper species, this species is a protogynous hermaphrodite: the fish begin life as females and then later transform into males when they have reached a larger size and matured (Sadovy and Colin, 1995; 
Bhandari et al., 2003). The other problem is that the survival of artificially reared larvae has remained low because of the high mortality rate at early developmental stages (Hirata et al., 2009). In Korea, the artificial seed production technique has been investigated since 1993, and small-scale seed production has been established since 2005 (Song et al., 2005; Lee et al., 2008). In addition, juvenile seedlings are being released for resource restoration. The development of appropriate markers is essential to preserve genetic diversity in wild populations and to monitor the diversity of culture stocks. However, such markers are not available for this species, in contrast to other grouper species (Stevenson et al., 1998; Zhao et al., 2009).

Table 3. Cross-species amplification in related Epinephelus species using the E. brunetus microsatellite loci.

\begin{tabular}{|c|c|c|c|c|}
\hline \multirow[t]{2}{*}{ Locus } & \multicolumn{4}{|c|}{ Genus: Epinephelus } \\
\hline & $\begin{array}{c}\text { E. brunetus } \\
71\end{array}$ & $\begin{array}{c}\text { E. septemfasciatus } \\
24\end{array}$ & $\begin{array}{l}\text { E. moara } \\
5\end{array}$ & $\begin{array}{l}\text { E. fuscoguttatus } \\
5\end{array}$ \\
\hline Eb01-nfrdi & $258-266$ & - & - & - \\
\hline Eb02-nfrdi & $134-138$ & - & 124 & - \\
\hline Eb05-nfrdi & $228-238$ & 224 & $222-224$ & $236-262$ \\
\hline Eb06-nfrdi & $175-205$ & $189-199$ & $185-195$ & $195-247$ \\
\hline Eb08-nfrdi & $237-249$ & - & $237-239$ & $217-249$ \\
\hline Eb11-nfrdi & 164 & - & - & - \\
\hline Eb22-nfrdi & $194-212$ & 184-192 & 184-186 & 188 \\
\hline Eb24-nfrdi & $196-202$ & 184-198 & $188-190$ & $202-246$ \\
\hline Eb27-nfrdi & $201-205$ & $219-247$ & 219 & $227-247$ \\
\hline Eb28-nfrdi & $231-281$ & $249-291$ & $255-277$ & $235-267$ \\
\hline Eb32-nfrdi & $163-173$ & $159-209$ & $155-157$ & $173-197$ \\
\hline Eb33-nfrdi & $244-250$ & - & 236 & 236 \\
\hline Eb40-nfrdi & 142 & $146-186$ & 142 & $122-140$ \\
\hline Eb42-nfrdi & $213-227$ & $203-211$ & 229 & $213-215$ \\
\hline Eb45-nfrdi & $186-242$ & $184-212$ & $196-202$ & $194-236$ \\
\hline Eb46-nfrdi & 205 & - & - & - \\
\hline Eb47-nfrdi & 195 & - & $197-217$ & $211-217$ \\
\hline Eb49-nfrdi & $236-238$ & 230 & $244-262$ & $248-282$ \\
\hline Eb50-nfrdi & $151-175$ & $139-153$ & $147-171$ & $153-183$ \\
\hline Eb51-nfrdi & 137 & $143-159$ & 143 & $169-193$ \\
\hline Eb52-nfrdi & $130-136$ & - & $130-132$ & $142-162$ \\
\hline Eb56-nfrdi & $160-168$ & $170-198$ & $162-164$ & 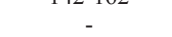 \\
\hline Eb57-nfrdi & $132-152$ & $118-120$ & $142-152$ & $138-154$ \\
\hline Eb58-nfrdi & $229-233$ & - & 143 & $233-235$ \\
\hline Eb59-nfrdi & $211-215$ & 209 & $221-231$ & $223-225$ \\
\hline Eb60-nfrdi & $138-172$ & - & $152-164$ & $144-180$ \\
\hline Eb63-nfrdi & $130-134$ & - & $132-152$ & - \\
\hline Eb64-nfrdi & $148-154$ & - & $144-164$ & - \\
\hline
\end{tabular}

In this study, we employed NGS techniques to develop microsatellite markers for $E$. bruneus. In addition to its cost- and time-effectiveness, NGS can produce a large number of candidates for selection. For example, only 63 loci from 66,452 SSRs containing dinucleotide repeats $(0.1 \%)$ were selected for initial testing, and the final 30 sets were selected based on their performance and annealing temperature $\left(60^{\circ} \mathrm{C}\right)$, which make multiplex PCR easy.

The number of alleles and allelic richness ranged from 2 to 23 and 2.0 to 22.8, respectively, which are similar to those that were reported for other Epinephelus species. Among the 23 loci tested, only three showed significant deviation from HWE, showing an excess of homozygotes. Although there are several reasons for this deviation, including inbreeding and the small population and sample sizes, the main reason may be the presence of null alleles (Vadopalas et al., 2004). In fact, among the three loci that showed deviation from HWE, two had null alleles. Considering their protogynous hermaphroditic nature and the fact that 
groupers form spawning aggregations, the maintenance of HWE in the analyzed samples is promising. It is well known that groupers sometimes form spawning aggregations with males derived from females of the same or a nearby aggregation, which can result in homozygosity and genetic substructure. For instance, evident population genetic structure was detected in the camouflage grouper (Epinephelus polyphekadion) in the Western Central Pacific, implicating limited larval dispersal and cohesion among cohorts within aggregations (Rhodes et al., 2003).

Although it is known that E. bruneus is widely distributed in the oceans of Eastern Asia, detailed genetic structures and gene flow among populations, which could explain the equilibrium, are not known, and future studies with the microsatellite markers developed here will be useful for that purpose.

One other advantage of the large number of SSR candidates is the higher probability of cross-species transfer of microsatellite markers. Cross-species transfer of microsatellite markers in the Epinephelus genus has been reported previously, but the number of loci is limited. For example, Zhao et al. (2009) successfully transferred 12 microsatellite markers of Epinephelus awoara to E. septemfasciatus and Centropristis striata. In contrast, a series of microsatellites developed for Epinephelus merra showed reliable amplification and variability in five additional epinephelines, but they proved extremely difficult to amplify in Epinephelus quernus (Nugroho et al., 1998). The five loci that were monomorphic in E. bruneus showed $60 \%$ transferability and polymorphism in three related species. Among these three species, E. moara showed the highest transferability and polymorphism, followed by E. fuscoguttatus and E. septemfasciatus. The transferability of microsatellite makers is known to be related to their taxonomic level (Barbará et al., 2007). In addition to their application to analyze genetic structure and diversity, the microsatellite makers can be used to identify species. This is especially valuable in grouper species. Generally, groupers are identified by their color patterns, body configuration, size, and number of body parts. However, the juveniles of many grouper species have color patterns that may be completely different from those of adults of the same species (Heemstra and Randall, 1993). Hence, identification based on morphology should be supported by other techniques, including the use of microsatellite markers. For example, Koedprang et al. (2007) identified eight grouper species using six microsatellite makers developed from Epinephelus malabaricus. The Eb01-nfrdi, Eb11-nfrdi, and Eb46-nfrdi loci, which are monomorphic only in E. bruneus, can be used to identify these species, and combinations of several loci can be used for species identification.

The economically important but vulnerable E. bruneus should be protected, and it must be produced in a sustainable manner by means of resource management and aquaculture. The microsatellite markers developed in this study could be used for that purpose in the future.

\section{ACKNOWLEDGMENTS}

Research supported by grants from the National Fisheries and Development Institute (NFRDI) in Korea.

\section{REFERENCES}

Barbará T, Palma-Silva C, Paggi GM, Bered F, et al. (2007). Cross-species transfer of nuclear microsatellite markers: potential and limitations. Mol. Ecol. 16: 3759-3767. 
Bhandari RK, Komuro H, Nakamura S, Higa M, et al. (2003). Gonadal restructuring and correlative steroid hormone profiles during natural sex change in protogynous honeycomb grouper (Epinephelus merra). Zool. Sci. 20: 1399-1404.

Chan WL (1968). Marine Fishes of Hong Kong. Part I, Hong Kong Govt. Press, Hong Kong, 128.

Chistiakov DA, Hellemans B and Volckaert FAM (2006). Microsatellites and their genomic distribution, evolution, function and applications: A review with special reference to fish genetics. Aquaculture 255: 1-29.

Choi Y, Kim JH and Park JY (2002). Marine Fishes of Korea. Kyo-Hak Publishing, Seoul, 267.

Greenley AP, Muguia-Vega A, Saenz-Arroyo A and Micheli F (2011). New tetranucleotide microsatellite loci in pink abalone (Haliotis corrugata) isolated via 454 pyrosequencing. Conserv. Genet. Resour. 4: 265-268.

Hamilton MB, Pincus EL, Di FA and Fleischer RC (1999). Universal linker and ligation procedures for construction of genomic DNA libraries enriched for microsatellites. Biotechniques 27: 500-507.

Heemstra PC and Randall JE (1993). FAO Species Catalogue. Groupers of the world (Family Serranidae, Subfamily Epinephelinae). An annotated and illustrated catalogue of the grouper, rockcod, hind, coral grouper and lyretail species known to date. Rome: FAO. FAO Fish. Synopsis 125: 382.

Hirata Y, Hamasaki K, Teruya K and Mushiake K (2009). Ontogenetic changes of body density of larvae and juveniles in seven-band grouper Epinephelus septemfasciatus and kelp grouper Epinephelus bruneus. Nippon Suissan Gakkaishi 75: 652-660.

Jarne P and Lagoda PJL (1996). Microsatellites, from molecules to populations and back. Trends Ecol. Evol. 11: $424-429$.

Kang JH, Park JY and Jo HS (2012). Rapid development of microsatellite markers with 454 pyrosequencing in a vulnerable fish, the mottled skate, Raja pulchra. Int. J. Mol. Sci. 13: 7199-7211.

Kato K, Ishimaru K, Sawada Y, Mutsuro J, et al. (2004). Ontogeny of digestive and immune system of larval and juvenile kelp grouper Epinephelus bruneus reared in the laboratory. Fish. Sci. 70: 1061-1069.

Kircher M and Kelso J (2010). High-throughput DNA sequencing-concepts and limitations. Bioessays 32: $524-536$.

Koedprang W, Na-Nakorn U, Nakajima M and Taniguchi N (2007). Evaluation of genetic diversity of eight grouper species Epinephelus spp. based on microsatellite variations. Fish. Sci. 73: 227-236.

Lee YD, Song YB, Lim BS and Oh SR (2008). Grouper aquaculture research in Jeju Island, Korea. Bull. Mar. Environ. Res. Inst. 32: 49-60.

Marte CL (2003). Larviculture of marine species in Southeast Asia: current research and industry prospects. Aquaculture 227: 293-304.

Nugroho E, Takagi M, Sugama K and Taniguchi N (1998). Detection of GT repeats microsatellite loci and their polymorphism for grouper of the genus Epinephelus. Fish. Sci. 64: 836-837.

Rhodes KL, Lewis RI, Chapman RW and Sadovy Y (2003). Genetic structure of camouflage grouper, Epinephelus polyphekadion (Pisces: Serranidae), in the western central Pacific. Mar. Biol. 142: 771-776.

Saarinen EV and Austin JD (2010). When technology meets conservation: increased microsatellite marker production using 454 genome sequencing on the endangered Okaloosa Darter (Etheostoma okaloosae). J. Hered. 101: 784-788.

Sadovy Y and Colin PL (1995). Sex development and sexuality in the Nassau grouper. J. Fish. Biol. 46: 961-976.

Sawada Y, Kato K, Okada T, Kurata M, et al. (1999). Growth and morphological development of larval and juvenile Epinephelus bruneus (Perciformes: Serranidae). Itchthyol. Res. 46: 245-257.

Song YB, Oh SR, Seo JP, Ji BG et al. (2005). Larval development and rearing of longtooth grouper Epinephelus bruneus in Jeju Island, Korea. J. World Aquaculture Soc. 36: 209-216.

Stevenson DE, Chapman RW and Sedberry GR (1998). Stock Identification in Nassau Grouper, Epinephelus striatus, Using Microsatellite DNA Analysis. Proceedings of the 50th Gulf and Caribbean Fisheries Institute, St. Croix, 727-749.

Thierry C, Sadovy Y and To AWL (2008). Epinephelus bruneus. In: IUCN 2012. IUCN Red List of Threatened Species. Version 2012.2. Available at [www.iucnredlist.org]. Accessed February 19, 2013.

Vadopalas B, Leclair LL and Bentzen P (2004). Microsatellite and allozyme analyses reveal few genetic differences among spatially distinct aggregations of geoduck clam (Panopea abrupta, Conrad 1849). J. Shellfish Res. 23: 693-706.

Zane L, Bargelloni L and Patarnello T (2002). Strategies for microsatellite isolation: a review. Mol. Ecol. 11: 1-16.

Zhao L, Shao CW, Liao XL, Ma HG et al. (2009). Twelve novel polymorphic microsatellite loci for the Yellow grouper (Epinephelus awoara) and cross-species amplifications. Conserv. Genet. 10: 743-745. 